\title{
Sarcopenia in older people with chronic airway diseases: the Rotterdam study
}

\author{
Elizabeth Benz ${ }^{1,2}$, Katerina Trajanoska ${ }^{1,2}$, Josje D. Schoufour ${ }^{1,2}$, Lies Lahousse $\mathbb{1}^{2,3}$, \\ Emmely W. de Roos (10 2,4, Natalie Terzikhan ${ }^{2,4}$, Carolina Medina-Gomez ${ }^{1}$, \\ Katia Verhamme ${ }^{5}$, Ross Williams $\mathbb{1}^{5}$, Bruno H. Stricker ${ }^{2,6}$, Oscar H. Franco ${ }^{2,7}$, \\ M. Arfan Ikram ${ }^{2,8}$, Fernando Rivadeneira ${ }^{1,2}$ and Guy Brusselle $\mathbb{1}^{2,4,6}$
}

Affiliations: 'Dept of Internal Medicine, Erasmus MC, University Medical Center Rotterdam, Rotterdam, The Netherlands. ${ }^{2}$ Dept of Epidemiology, Erasmus MC, University Medical Center Rotterdam, Rotterdam, the Netherlands. ${ }^{3}$ Dept of Bioanalysis, FFW, Ghent University, Ghent, Belgium. ${ }^{4}$ Dept of Respiratory Medicine, Ghent University Hospital, Ghent University, Ghent, Belgium. ${ }^{5}$ Dept of Medical Informatics, Erasmus MC, University Medical Center Rotterdam, Rotterdam, the Netherlands. ${ }^{6}$ Dept of Respiratory Medicine Erasmus MC, University Medical Center Rotterdam, Rotterdam, the Netherlands. ${ }^{7}$ Institute of Social and Preventive Medicine, University of Bern, Bern, Switzerland. ${ }^{8}$ Dept of Neurology, Erasmus MC, University Medical Center Rotterdam, Rotterdam, the Netherlands.

Correspondence: Guy Brusselle, Dept of Epidemiology, Erasmus MC, University Medical Center Rotterdam, P.O. Box 2040, 3000 CA, Rotterdam, the Netherlands. E-mail: g.brussellederasmusmc.nl

ABSTRACT Sarcopenia is a heterogeneous skeletal muscle disorder involving the loss of muscle mass and function. However, the prevalence of sarcopenia based on the most recent definition remains to be determined in older people with chronic airway diseases.

The aim was to evaluate sarcopenia prevalence and association with chronic airway diseases and its lung function in an older population, using the European Working Group on Sarcopenia in Older People 2 (EWGSOP2) criteria.

We performed a cross-sectional analysis in 5082 participants (mean age $69.0 \pm 8.8$ years, $56 \%$ females) from the Rotterdam Study. Participants with interpretable spirometry and an available assessment of sarcopenia were included. The appendicular skeletal muscle mass index (ASMI) and handgrip strength (HGS) were assessed using dual-energy X-ray absorptiometry (DXA) and a hydraulic hand dynamometer, respectively. We analysed the association between sarcopenia and chronic airway diseases by using regression models adjusted for age, sex, smoking status, total fat percentage and other relevant confounders.

Participants with chronic airway diseases had higher prevalence of probable sarcopenia $(12.0 \%, 95 \%$ CI 10.2-13.8) and confirmed sarcopenia (3.0\%, 95\% CI 2.1-3.9) than without. Chronic airway diseases were associated with "probable sarcopenia" (OR 1.28, 95\% CI 1.02-1.60), "confirmed sarcopenia" (OR 2.13, 95\% CI 1.33-3.43), reduced HGS $(\beta-0.51(-0.90-0.11))$ and reduced ASMI $(\beta-0.19(-0.25--0.14))$. Forced expiratory volume in $1 \mathrm{~s}<80 \%$ was associated with lower HGS $(\beta-1.03(-1.75--0.31))$ and lower ASMI $(\beta-0.25(-0.36--0.15))$ than forced expiratory volume in $1 \mathrm{~s} \geqslant 80 \%$.

Sarcopenia was prevalent and associated with chronic airway diseases among older population. These results suggest the need for early diagnosis of sarcopenia in older people with chronic airway diseases by applying EWGSOP2 recommendations.

@ERSpublications

Probable and confirmed sarcopenia prevalence among the older population with chronic airway diseases is higher than in those without. Given this prevalence, it could be essential to screen for sarcopenia in primary care units for older patients. https://bit.ly/34Fhc56

Cite this article as: Benz E, Trajanoska K, Schoufour JD, et al. Sarcopenia in older people with chronic airway diseases: the Rotterdam study. ERJ Open Res 2021; 7: 00522-2020 [https://doi.org/ $10.1183 / 23120541.00522-2020]$.

This article has supplementary material available from openres.ersjournals.com

Received: 13 Aug 2020 | Accepted: 28 Sept 2020

Copyright CERS 2021. This article is open access and distributed under the terms of the Creative Commons Attribution Non-Commercial Licence 4.0. 


\section{Introduction}

Changes in muscles undergo permanent shifts, particularly after the 5 th decade of life, there is $1-2 \%$ loss of muscle mass per year [1] and 1.5-5\% loss of muscle strength [2]. This condition is called sarcopenia [3], which affects around $10 \%$ of the healthy older population [4]. Nevertheless, sarcopenia is not only a problem in older people, but it is also a consequence of chronic diseases [3]. Recently, the European Working Group on Sarcopenia in Older People (EWGSOP2) has defined secondary sarcopenia as a consequence of concomitant long-term conditions, such as chronic airway diseases (i.e. chronic obstructive pulmonary disease (COPD)) [5].

Asthma, COPD and asthma-COPD overlap (ACO) are the leading chronic airway diseases that encompass multiple clinical phenotypes and molecular endotypes with significant differences in clinical, functional, imagining, prognosis and response to treatment [6, 7]. Around 30\% of the global population is affected by asthma during their lifetime [8], and $10 \%$ of the adult population has COPD [9, 10]. ACO is a syndrome that affects between $9 \%$ and $55 \%$ of those with asthma or COPD [8]. Attention for these chronic airway diseases is rising, given their increasing burden related to multiple extrapulmonary manifestations which gradually contribute to disability $[7,11]$. Notably, in COPD patients, the evidence is emerging that sarcopenia affects functional capacity [11]. The prevalence of sarcopenia in COPD patients has been reported to range widely, from $8 \%$ to $63 \%$, depending on the adopted diagnostic method, definition criteria and the population setting [12]. In the last decade, we have broadened our understanding of the pathophysiology of primary sarcopenia in healthy older adults. In contrast, the mechanisms leading to secondary sarcopenia (in patients with chronic health conditions) remain unclear; mainly because there are multiples common features linked with both sarcopenia and COPD, such as age, physical inactivity, weight loss, lung function, smoking, inflammatory cytokines and comorbidities, among others [13-15].

Although the association between sarcopenia and COPD population has been reported in the past $[16,17]$, there are no studies that have evaluated this association using the new consensus definition and cut-off values recommended by EWGSOP2. Therefore, we aimed to provide an up-to-date estimate of the prevalence of sarcopenia among older people with chronic airway diseases by applying the EWGSOP2 criteria. Our secondary objective was to determine the association between sarcopenia (probable and confirmed) and its components (handgrip and appendicular skeletal muscle index), and chronic airway diseases and lung function parameters.

\section{Methods}

\section{Study design and participants}

Our cross-sectional analysis was conducted within the Rotterdam Study, an ongoing prospective population-based study, that investigates the occurrence and risk factors for chronic diseases in older people $[6,18]$. The details of the study objectives, design and main findings have been recently updated [6]. Briefly, the Rotterdam Study started in 1990 and has approximately 15000 participants enrolled in three cycles (RSI, RSII and RSIII) from the Ommoord district, a well-defined suburb of the city of Rotterdam (the Netherlands) [6]. Every 4-5 years, participants undergo a home interview and a visit to the research centre for clinical examination and tests (e.g. spirometry). We included all participants with interpretable spirometry and reliable measurements of handgrip strength (HGS) and lean mass by DXA, who visited the research centre between 2009 and 2014 (Supplementary figure 1). Other relevant data in this study were retrieved from medical records from the general practitioners and prescription data from pharmacies serving the Ommoord area. The Rotterdam Study was approved by the medical ethics committee of the Erasmus Medical Center, Rotterdam (registration number MEC 02.1015), and by the Dutch Ministry of Health, Welfare and Sport (Population Screening Act WBO, license number 1071272-159521-PG). All participants provided written informed consent.

\section{Assessments}

Assessment of chronic airways diseases

Trained paramedic personnel performed pre-bronchodilator spirometry using a Master Screen PTF Pro spirometer (CareFusion, Houten, the Netherlands) according to the American Thoracic Society (ATS) and the European Respiratory Society (ERS) guidelines [19]. Predicted values of forced expiratory volume during in $1 \mathrm{~s}\left(\mathrm{FEV}_{1)}\right.$ and forced vital capacity $(\mathrm{FVC})$ were calculated using the reference equations obtained from the Global Lung Function Initiative (GLI) which takes into account age, sex, height and ethnicity [20]. Severity of obstruction in COPD was determined based on the modified Global Initiative for Chronic Obstructive Lung Disease (GOLD) criteria: $\mathrm{FEV}_{1} \geqslant 80 \%$ predicted; without or mild airflow limitation and $\mathrm{FEV}_{1}<80 \%$ predicted; moderate-to-severe airflow limitation [10]. The same cut-off values were used to represent severity of obstruction in asthma and ACO cases. 
We considered participants diagnosed with chronic airways diseases before or at the time of DXA scan. Chronic airway diseases included participants with asthma, COPD and ACO. COPD cases were identified by spirometry data $\left(\mathrm{FEV}_{1} / \mathrm{FVC}\right.$ ratio $\left.<0.7\right)$ and medical records. Asthma cases were defined based on physician-diagnosed asthma, as evidenced in the medical records [21]. ACO cases were defined as asthmatic participants who met the obstructive spirometry definition of COPD.

Participants who had restrictive spirometry or restrictive disease were classified as other lung diseases. Participants who did not meet the definition of asthma or COPD because they had no clinical diagnosis or normal spirometry $\left(\mathrm{FEV}_{1} / \mathrm{FVC} \geqslant 0.7\right.$ and $\left.\mathrm{FVC} \geqslant 80 \%\right)$ at the time of the DXA scan date, were considered as subjects without chronic airway diseases.

\section{Assessment of sarcopenia}

Sarcopenia status was defined using the EWGSOP2 criteria (table 1) [5]. Participants with low handgrip were classified as having "probable sarcopenia". Those with low handgrip and low lean mass were defined as having "confirmed sarcopenia". And those with "confirmed sarcopenia" plus low gait speed were considered as having "severe sarcopenia".

A single trained examinator performed handgrip test using a hydraulic hand dynamometer (Fabrication Enterprises Inc., White Plains, NY, USA). Maximum HGS was obtained as the maximum value (kg) out of three trials performed in the non-dominant hand [6]. Low HGS was defined as $<27 \mathrm{~kg}$ for males and $<16 \mathrm{~kg}$ for females [5]. Lean mass was measured by DXA scan, using an iDXA total body-beam densitometer (GE Lunar Corp, Madison, WI, USA). The scans were analysed with enCORE software V13.6 providing measurements across pre-defined body regions of interest, namely the head, trunk, arms and legs. The sum of the lean mass from the upper and lower limbs is called appendicular lean mass (ALM). Appendicular skeletal muscle mass index (ASMI) was defined as ALM divided by the height squared $\left(\mathrm{m}^{2}\right)$. Low lean mass was classified as an ASMI of $<7.0 \mathrm{~kg} \cdot \mathrm{m}^{-2}$ for males and $<5.5 \mathrm{~kg} \cdot \mathrm{m}^{-2}$ for females [5]. Another trained examinator measured gait speed during normal walk using the GAITRite system (GAITRite Platinum; CIR Systems Inc., Sparta, NJ, USA) in a $5.79 \mathrm{~m}$ long electronic walkway with $1.27 \mathrm{~cm}$ wide pressure sensors [22]. Low gait speed was defined as $\leqslant 0.8 \mathrm{~m} \cdot \mathrm{s}^{-1}$ for females and males [5].

\section{Assessment of covariables}

Body mass index (BMI) calculated as weight $(\mathrm{kg})$ divided by height squared $\left(\mathrm{kg} \cdot \mathrm{m}^{-2}\right)$ was used to classify overweight (BMI $\left.25-30 \mathrm{~kg} \cdot \mathrm{m}^{-2}\right)$ and obesity $\left(B M I \geqslant 30 \mathrm{~kg} \cdot \mathrm{m}^{-2}\right)$. Age was categorised into two groups $(<70$ and $\geqslant 70$ years). Smoking status (never, past and current) was collected during home interviews. Total body fat percentage was calculated as total body fat mass $(\mathrm{kg})$ divided by body weight $(\mathrm{kg})$ multiplied by 100. Physical activity was assessed by a validated adapted version of the Longitudinal Aging Study Amsterdam (LASA) Physical Activity Questionnaire (LAPAQ) [23] and expressed in metabolic equivalent of task (MET)-hours per week. Protein intake percentage was calculated using the formula: $4 \times$ protein intake g)/daily total energy $(\mathrm{kcal}) \times 100$. Participants who had no oral corticosteroid prescriptions (Anatomical Therapeutic Chemical code (ATC): H02) before the DXA scan date were defined as never users. Then we categorised users into current users when their last prescription was recorded $\leqslant 90$ days before DXA scan, and past users when their last prescription was recorded $>90$ before DXA scan.

We identified four prevalent comorbid conditions before or at the time of DXA scan for each participant. 1) Type 2 diabetes (fasting plasma glucose level $\geqslant 7 \mathrm{mmol} \cdot \mathrm{L}^{-1}$, or a non-fasting plasma glucose level $\geqslant 11.1 \mathrm{mmol} \cdot \mathrm{L}^{-1}$ or the use of blood glucose-lowering medication) [24]; 2) hypertension (systolic blood pressure $\geqslant 160 \mathrm{mmHg}$ or diastolic blood pressure $\geqslant 100 \mathrm{mmHg}$ or use of antihypertensive medication) [25];

TABLE 1 Definition of sarcopenia and cut-off values based on the revised and updated EWGSOP2 consensus

\begin{tabular}{|c|c|c|c|}
\hline \multirow[t]{2}{*}{ Definition } & \multirow[t]{2}{*}{ Outcome } & \multicolumn{2}{|c|}{ Cut-off values } \\
\hline & & Male & Female \\
\hline \multirow[t]{2}{*}{$\begin{array}{l}\text { Probable sarcopenia } \\
\text { Sarcopenia }\end{array}$} & $\begin{array}{l}\text { Low muscle strength: measured by hydraulic hand dynamometer } \\
\text { Low muscle strength AND }\end{array}$ & $<27 \mathrm{~kg}$ & $<16 \mathrm{~kg}$ \\
\hline & Low lean mass: measured by DXA & $<7.0 \mathrm{~kg} \cdot \mathrm{m}^{-2}$ & $<5.5 \mathrm{~kg} \cdot \mathrm{m}^{-2}$ \\
\hline \multirow[t]{2}{*}{ Severe sarcopenia } & Low muscle strength AND low lean mass AND & & \\
\hline & $\begin{array}{l}\text { Low physical performance: measured by gait speed or } \\
\text { Timed Up and Go Test }\end{array}$ & $\begin{array}{l}\leqslant 0.8 \mathrm{~m} \cdot \mathrm{s}^{-1} \\
\quad \geqslant 20 \mathrm{~s}\end{array}$ & $\begin{array}{l}\leqslant 0.8 \mathrm{~m} \cdot \mathrm{s}^{-1} \\
\quad \geqslant 20 \mathrm{~s}\end{array}$ \\
\hline
\end{tabular}


3) coronary heart disease (myocardial infarction, coronary artery bypass grafting (CABG) or percutaneous coronary intervention (PCI) [26]; and 4) cancer (according to the International Classification of Diseases, 10th revision (ICD-10)) [27].

\section{Statistical analyses}

Descriptive characteristics were expressed as mean $\pm \mathrm{SD}$ or as median (interquartile range (IQR) and as frequency (\%). Data comparisons between participants with and without chronic airway diseases were studied using t-test, Chi-squared test and multiple proportion Chi-squared test. Prevalence of sarcopenia was calculated by dividing the total number of cases by the total number of participants and $95 \%$ confidence intervals. Comparisons of sarcopenia status prevalence in these categories were tested using Chi-squared tests.

Association between chronic airway diseases and sarcopenia status was examined by multinomial logistic regression adjusted for previously identified confounders [13-15, 28, 29] such as age, sex, percentage of fat, height and smoking. Linear regression models adjusted for the aforementioned confounders were used to investigate the association between chronic airway diseases and the individual components of sarcopenia. Moreover, in chronic airway diseases, we assessed the association between sarcopenia (and its components) and $\mathrm{FEV}_{1} \geqslant 80 \%$ versus $\mathrm{FEV}_{1}<80 \%$ by using logistic and linear models adjusted for confounders mentioned above. We did separate sensitivity analyses, including participants without comorbidities and exploring the association between $\mathrm{FEV}_{1}$ (\% predicted) and sarcopenia. Moreover, we performed additional adjustments for $\mathrm{FEV}_{1} / \mathrm{FVC}$, oral corticosteroids use, comorbidities, physical activity and percentage of protein intake that might be either confounders or mediators.

For data missing more than $5 \%$, we used the multiple imputation technique by chained equations. We reported only pooled effect results in the regression models. P-values of less than 0.05 were considered significant. All statistical analyses were performed using SPSS (IBM SPSS Statistics 24) and R version 3.6.0 (2019-04-26).

\section{Results}

Main characteristics of the study population

Among 7162 participants from the Rotterdam Study, 5306 had interpretable lung function and reliable assessments of sarcopenia between 2009-2014. Of these, 224 participants were excluded due to other lung diseases. Finally, 5082 participants (71.0\%) were analysed in this study. 1246 participants had chronic airway diseases, and 3836 had not (figure 1). Among these, 779 (62.5\%) had COPD, 439 (35.2\%) had asthma, and $28(2.3 \%)$ had ACO.

The mean \pm SD age of the study population was $69.0 \pm 8.8$ years, and $56.3 \%(n=2863)$ of them were female. Study population characteristics are presented in table 2.

Participants with chronic airway diseases were older, were significantly more often past or current smokers $(p<0.001)$, had lower lung function $(p<0.001)$, lower handgrip strength $(p<0.001)$, and were more frequently oral corticosteroids users $(\mathrm{p}<0.001)$ than those without. Moreover, they presented with a higher prevalence of comorbidities such as diabetes mellitus (16.8\%), hypertension (75.5\%) and coronary heart diseases $(11.9 \%)$ than without. Sarcopenia status stratified by the chronic airway diseases is described in supplementary table 1 .

\section{Prevalence of "probable sarcopenia" and "confirmed sarcopenia"}

The overall prevalence of sarcopenia (probable and confirmed) was 11.6\% (95\% CI 11.1-12.9) and increased with age (supplementary figure 2) and number of comorbidities (supplementary table 2). Participants older than 70 years had higher prevalence of probable sarcopenia (18.3\% (95\% CI 16.4-19.6)) and confirmed sarcopenia $(3.0 \%$ (95\% CI 2.2-3.7)) than younger than 70 years $(3.5 \%$ (95\% CI 3.3-4.7) and $0.5 \%$ (95\% CI $0.2-0.7$ ) respectively) (supplementary figure 2). Males had higher prevalence of confirmed sarcopenia than females $(2.0 \%$ (95\% CI 1.4-2.6);1.0\% (95\% CI 0.6-1.4), respectively) (figure 2a). Ten participants had severe sarcopenia (mean age $80.9 \pm 6.4$ years) (supplementary table 3 ). To perform the association analyses, these participants were included as confirmed sarcopenia status.

Participants with chronic airway diseases had higher prevalence of probable sarcopenia (12.0\% (95\% CI 10.2-13.8), 150 cases) and confirmed sarcopenia (3.0\% (95\% CI 2.1-3.9), 36 cases) (figure 2b) than those without (9.3\%, (95\% CI 8.1-10.0) and 1.2\% (95\% CI 0.7-1.3) respectively). Prevalence of sarcopenia among each chronic airway diseases (i.e. COPD, asthma) is described in supplementary table 4. Males with chronic airway diseases had higher percentage of low handgrip and low ASMI than males without; whereas, females had higher percentage of low ASMI compared to females without chronic airway diseases (supplementary table 5). 


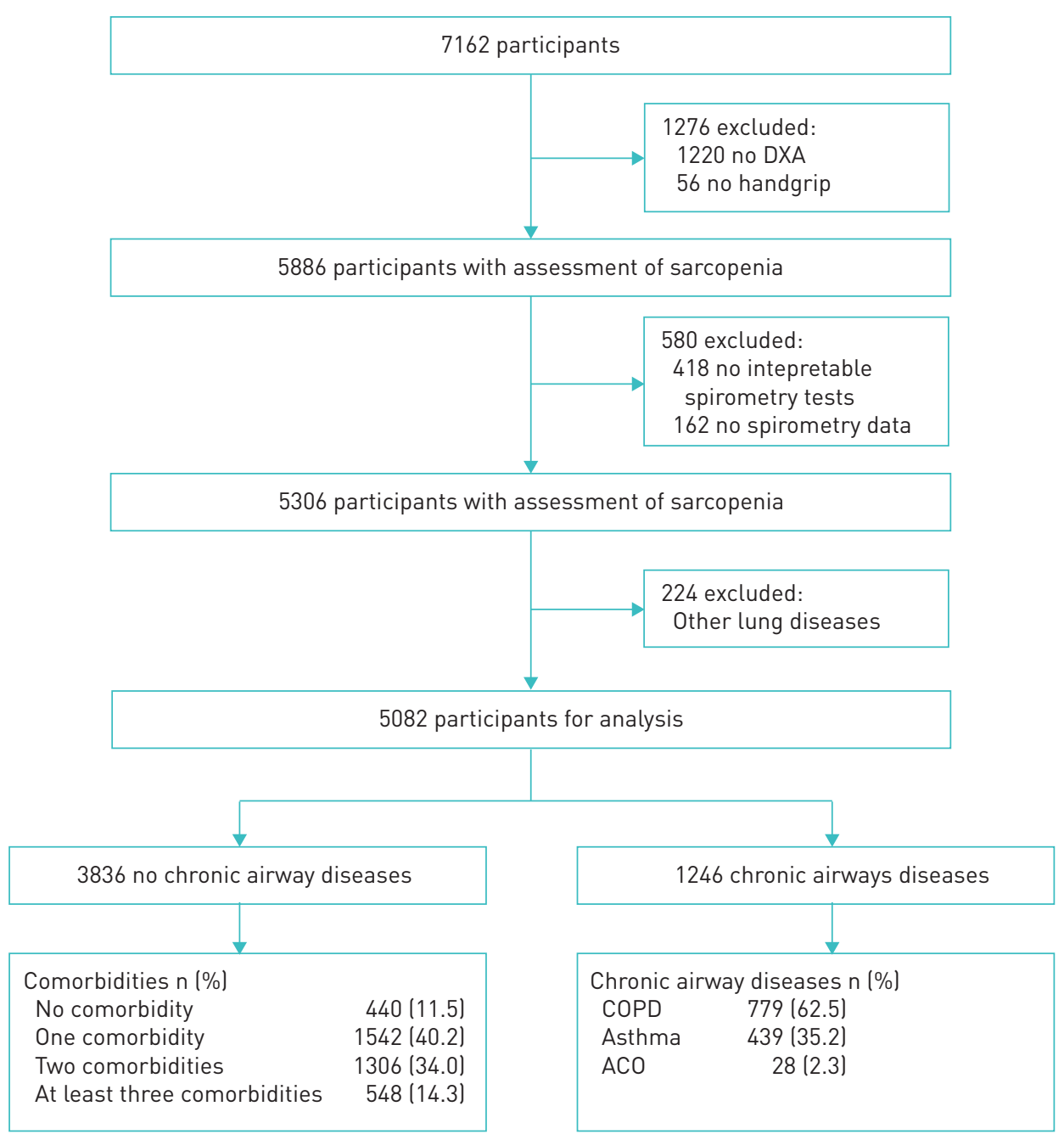

FIGURE 1 Flow chart of the study population. DXA: dual-energy X-ray absorptiometry; ACO: Asthma and COPD Overlap.

Association between chronic airways diseases and the prevalence of sarcopenia Participants with chronic airways diseases had higher odds of having probable sarcopenia (1.28 (95\% CI 1.02-1.60)) and two times higher odds to have confirmed sarcopenia (2.13 (95\% CI 1.33-3.43)) than participants without (table 3).

Association between chronic airway diseases and the components of sarcopenia: handgrip strength and appendicular skeletal muscle index

Adjusted for age, sex, total body fat $(\%)$ and smoking, HGS $(\beta-0.51(-0.90,-0.11), \mathrm{p}<0.001)$ and ASMI $(\beta-0.19(-0.25,-0.14) ; \mathrm{p}<0.001)$ were lower in participants with chronic airway diseases compared to without (table 4).

\section{Association between lung function and sarcopenia (and its components)}

In participants with chronic airway diseases, $\mathrm{FEV}_{1}<80 \%$ was not significantly associated with sarcopenia (probable and confirmed) (Supplementary table 6 ). $\mathrm{FEV}_{1}<80 \%$ was associated with lower HGS $(\beta=-1.03$ $(-1.75 ;-0.31)$ and lower and ASMI $(\beta=-0.25(-0.36 ;-0.15))$ compared with those who had $\mathrm{FEV}_{1} \geqslant 80 \%$ (table 5).

\section{Sensitivity analysis}

When including only participants with no comorbidity $(\mathrm{n}=566)$, the prevalence of probable sarcopenia in no chronic airway diseases decreased from $23.9 \%$ to $4.2 \%$, and confirmed sarcopenia from $23.9 \%$ to $15.2 \%$ (supplementary table 7). There were only a few cases for participants with confirmed sarcopenia. Therefore, associations between sarcopenia and chronic airway diseases in this subgroup (without 


\begin{tabular}{|c|c|c|c|c|}
\hline Participants & $\begin{array}{c}\text { Total } \\
\text { population }\end{array}$ & $\begin{array}{c}\text { No chronic airway } \\
\text { diseases }\end{array}$ & $\begin{array}{c}\text { Chronic airway } \\
\text { diseases }\end{array}$ & p-value \\
\hline Subjects $\mathrm{n}$ & 5082 & 3836 & 1246 & \\
\hline Age years & $69.0 \pm 8.8$ & $68.6 \pm 8.5$ & $70.1 \pm 9.1$ & $<0.001$ \\
\hline Age groups & & & & $<0.001$ \\
\hline$<70$ years & 2873 (56.5) & 2234 (58.2) & 639 (51.3) & \\
\hline$\geqslant 70$ years & 2209 (43.5) & $1602(41.8)$ & 607 (48.7) & \\
\hline Sex & & & & $<0.001$ \\
\hline Male & 2219 (43.7) & $1620(42.2)$ & 599 (48.1) & \\
\hline Female & 2863 (56.3) & 2216 (58.8) & 647 (51.9) & \\
\hline BMI $\mathbf{k g} \cdot \mathrm{m}^{-2}$ & $27.5 \pm 4.2$ & $27.5 \pm 4.1$ & $27.2 \pm 4.4$ & 0.061 \\
\hline Overweight & $2446(48.1)$ & $1897(49.4)$ & 549 (44.1) & $<0.0029$ \\
\hline Obese & $1168(23.0)$ & $882(23.0)$ & 286 (22.9) & \\
\hline Smoking & & & & $<0.05$ \\
\hline Never & 1717 (33.8) & $1436(37.4)$ & $281(22.5)$ & \\
\hline Past & 2749 (54.1) & 2041 (53.2) & 708 (56.8) & \\
\hline Current & $616(12.1)$ & 359 (9.4) & $257(20.6)$ & \\
\hline \multicolumn{5}{|l|}{ Lung function } \\
\hline $\mathrm{FEV}_{1} / \mathrm{FVC} \%$ & $76.0 \pm 7.7$ & $78.8 \pm 4.6$ & $67.5 \pm 8.9$ & $<0.001$ \\
\hline FVC predicted \% & $99.5 \pm 14.6$ & $101.5 \pm 13.3$ & $93.3 \pm 16.5$ & $<0.001$ \\
\hline $\mathrm{FEV}_{1}$ predicted $\%$ & $98.5 \pm 17.8$ & $103.7 \pm 13.9$ & $82.5 \pm 19.1$ & $<0.001$ \\
\hline \multicolumn{5}{|l|}{ Total body fat $\%$} \\
\hline Male & $31.0 \pm 5.5$ & $30.9 \pm 5.3$ & $31.2 \pm 6.1$ & 0.292 \\
\hline Female & $40.1 \pm 6.1$ & $40.1 \pm 6.0$ & $39.9 \pm 6.5$ & 0.595 \\
\hline \multicolumn{5}{|l|}{ HGS kg } \\
\hline Male & $37.1 \pm 8.8$ & $37.4 \pm 8.6$ & $36.2 \pm 9.2$ & $<0.05$ \\
\hline Female & $22.1 \pm 5.7$ & $22.3 \pm 5.7$ & $21.5 \pm 6.0$ & $<0.05$ \\
\hline \multicolumn{5}{|l|}{ ASMI $\mathrm{kg} \cdot \mathrm{m}^{-2}$} \\
\hline Male & $8.3 \pm 0.9$ & $8.4 \pm 0.9$ & $8.0 \pm 0.9$ & $<0.001$ \\
\hline Female & $6.9 \pm 0.9$ & $6.9 \pm 0.8$ & $6.8 \pm 0.9$ & $<0.05$ \\
\hline Walking speed ${ }^{\#} \mathrm{~m} \cdot \mathrm{s}^{-1}$ & $1.2(1.1-1.3)$ & $1.2(1.1-1.4)$ & $1.2(1.1-1.3)$ & \\
\hline PA MET $^{\#} h \cdot$ week $^{-2}$ & $42.6(17.3-81.3)$ & $44.5(18.0-83.9)$ & $36.8(15.0-73.7)$ & \\
\hline Protein intake ${ }^{\#} \%$ & $15.7 \pm 2.9$ & $15.7 \pm 2.8$ & $15.5 \pm 3.0$ & \\
\hline $\mathrm{OCS}^{\#}$ & & & & $<0.001$ \\
\hline Never & $3364(66.2)$ & 2749 (71.7) & 615 (49.3) & \\
\hline Past & 1592 (31.3) & $1029(26.8)$ & $563(45.2)$ & \\
\hline Current & $126(2.5)$ & $58(1.5)$ & $68(5.5)$ & \\
\hline \multicolumn{5}{|l|}{ Comorbidities } \\
\hline Type 2 diabetes & 699 (13.8) & 489 (12.7) & 210 (16.8) & $<0.001$ \\
\hline Hypertension & 3656 (71.9) & $2715(70.8)$ & 941 (75.5) & $<0.05$ \\
\hline Cancer & $524(10.3)$ & $389(10.1)$ & $135(10.8)$ & 0.484 \\
\hline $\mathrm{CHD}$ & $434(8.5)$ & $286(7.5)$ & $148(11.9)$ & $<0.001$ \\
\hline \multicolumn{5}{|l|}{ Sarcopenia status } \\
\hline Probable sarcopenia & $506(10.0)$ & 356 (9.3) & $150(12.0)$ & $<0.001$ \\
\hline $\begin{array}{l}\text { Confirmed } \\
\text { sarcopenia }\end{array}$ & $82(1.6)$ & $46(1.2)$ & $36(3.0)$ & \\
\hline
\end{tabular}

Data are presented as mean $\pm \mathrm{SD}, \mathrm{n}(\%)$ or median (interquartile range), unless otherwise stated. BMI: body mass index (overweight: BMI $25-30 \mathrm{~kg} \cdot \mathrm{m}^{-2}$, obese: $B M I \geqslant 30 \mathrm{~kg} \cdot \mathrm{m}^{-2}$ ); $F E V_{1} / F V C$ : forced expiratory volume in $1 \mathrm{~s} /$ forced vital capacity ratio; HGS: handgrip strength; ASMI: appendicular skeletal muscle mass index; PA: physical activity; MET: metabolic equivalent of task; OCS: oral corticosteroids use; CHD: coronary heart diseases. ${ }^{\#}: \mathrm{n}(\%)$ of missing values per variable. Walking speed: 1428 (28.1); physical activity: 601 (11.8); protein intake: 1041 (20.5); diabetes mellitus: 92 (1.8); hypertension: 1 (0.0); CHD: 53 (1.0). Original data without imputations.

comorbidities) were not possible. In the linear regression models, handgrip was not associated with chronic airway diseases; whereas, appendicular skeletal muscle mass index remained associated (supplementary table 8).

Regarding the spirometric parameters, among chronic airway diseases, $\mathrm{FEV}_{1}$ (\% pred) was lower for all sarcopenic persons (probable and confirmed) (supplementary figure 3). Associations between the 


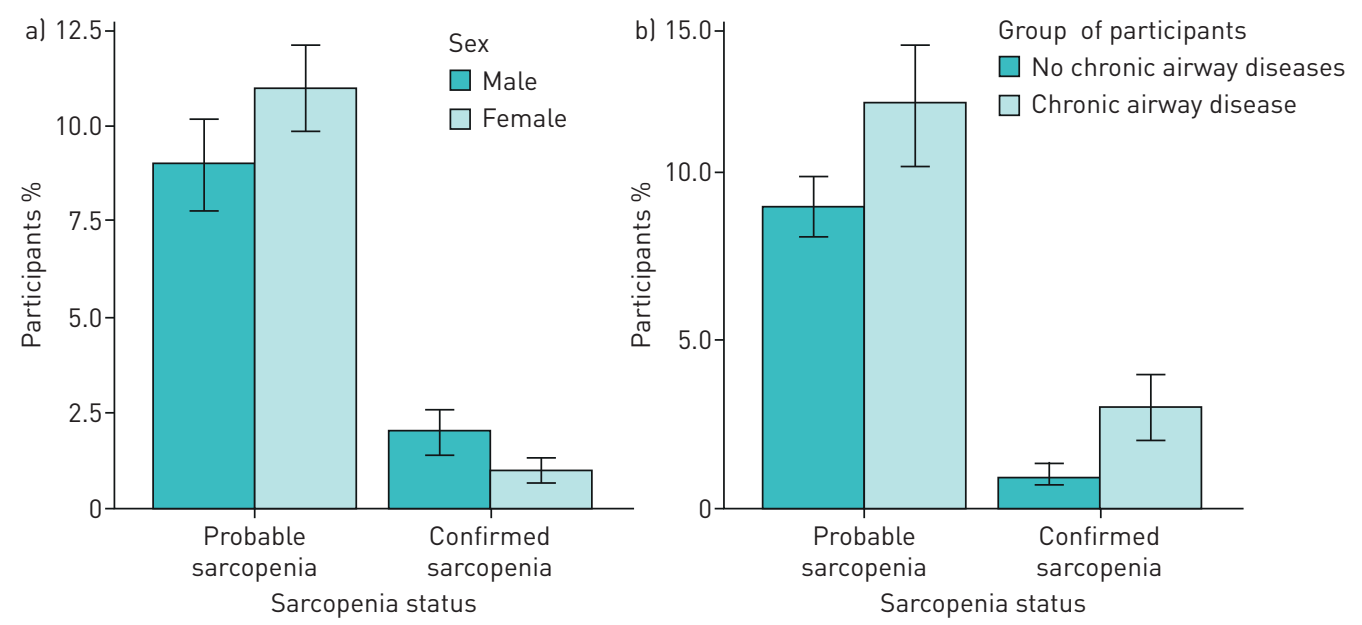

FIGURE 2 Prevalence of probable sarcopenia and confirmed sarcopenia according to a) sex and b) the presence or absence of chronic airway diseases.

components of sarcopenia and lung function in chronic airway disease participants are presented in supplementary material figure 4 and table 9. HGS and ASMI were associated with $\mathrm{FEV}_{1}$ (\% pred) independent of sex, age, total body fat $(\%)$ and smoking status $(\beta=0.04(0.03 ; 0.07)$ and $\beta=0.01(0.01$; 0.02) respectively). The associations between the spirometric parameters and the components of sarcopenia are investigated for participants without chronic airway diseases in supplementary material figure 4 and table 9 .

Models with additional adjustment for lung function ( $\left.\mathrm{FEV}_{1} / \mathrm{FVC}\right)$, oral corticosteroids use, comorbidities, physical activity and percentage of protein intake on the association between sarcopenia status and chronic airway diseases are presented in table 6.

\section{TABLE 3 Association between chronic airway diseases and sarcopenia (EWGSOP2)}

\begin{tabular}{|c|c|c|c|}
\hline Sarcopenia status & Chronic airway diseases & $\begin{array}{c}\text { Model } 1 \\
\text { OR }(95 \% \mathrm{CI})\end{array}$ & $\begin{array}{c}\text { Model } 2 \\
\text { OR (95\% CI) }\end{array}$ \\
\hline Probable sarcopenia $(n=506)$ & $\begin{array}{l}\text { No }(n=356) \\
\text { Yes }(n=150)\end{array}$ & $\begin{array}{c}\text { Ref. } \\
1.20(0.96-1.49)\end{array}$ & $\begin{array}{c}\text { Ref. } \\
1.28(1.02-1.60)\end{array}$ \\
\hline Confirmed sarcopenia ( $n=82$ ) & $\begin{array}{l}\text { No }(n=46) \\
\text { Yes }(n=36)\end{array}$ & $\begin{array}{c}\text { Ref. } \\
2.03(1.29-3.21)\end{array}$ & $\begin{array}{c}\text { Ref. } \\
2.13(1.33-3.43)\end{array}$ \\
\hline
\end{tabular}

EWGSOP2: European Working Group of Sarcopenia in Older People 2; Ref.: no chronic airway diseases. Model 1 was adjusted for age and sex; model 2 was model 1 plus total body fat $(\%)$, height $(\mathrm{cm})$ and smoking status.

TABLE 4 Association between chronic airway diseases and handgrip muscle strength (HGS) and appendicular skeletal muscle index (ASMI)

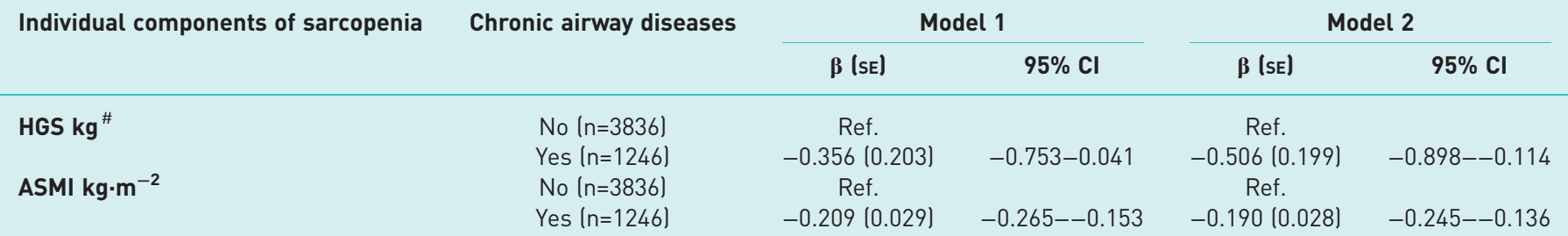

Ref.: no chronic airway diseases. Model 1 was adjusted for age and sex; model 2 was model 1 plus total body fat (\%), height (cm) and smoking status. " : height is only included in the HGS model due to ASMI already being calculated based on height $\left(\mathrm{m}^{2}\right)$. 


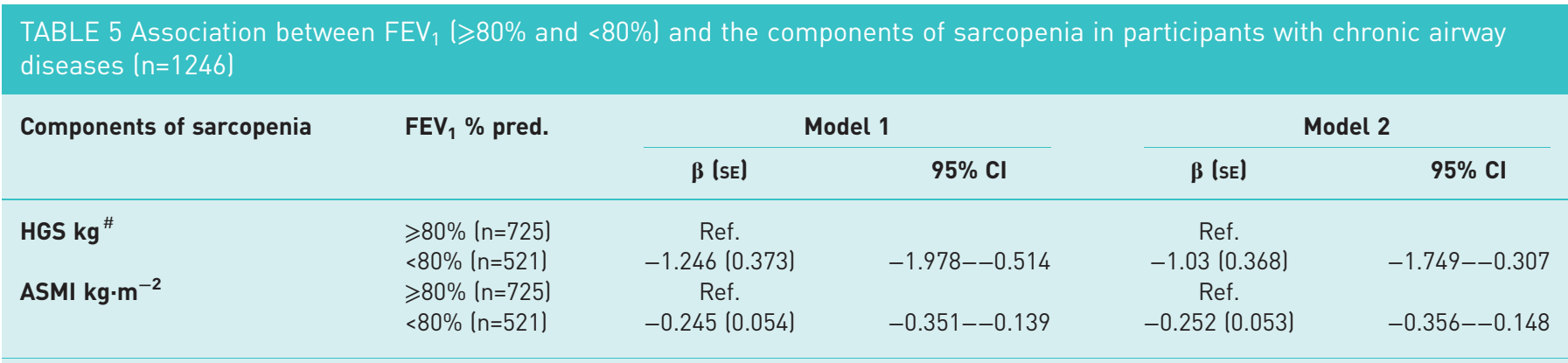

$\mathrm{FEV}_{1}$ : forced expiratory volume in $1 \mathrm{~s} ;$ HGS: handgrip strength; ASMI: appendicular skeletal muscle mass index. Model 1 was adjusted for age and sex; model 2 was model 1 plus total body fat (\%), height and smoking status. \#: height is only included in the HGS model due to ASMI already being calculated based on height $\left(\mathrm{m}^{2}\right)$.

\section{Discussion}

In this large population-based study of older people, the prevalence of probable sarcopenia (low handgrip) was higher in participants with $(12.0 \%)$ than without $(9.3 \%)$ chronic airway diseases. Likewise, confirmed sarcopenia (low handgrip and low lean mass) was higher in persons with chronic airway diseases (3.0\%) compared with those without (1.2\%). Sarcopenia (probable and confirmed) was associated with chronic airway disease independent of age, sex, total body fat, height and smoking status. Moreover, when examining the individual components of sarcopenia, we demonstrated that older persons with chronic airway diseases had both a lower appendicular lean mass index and lower handgrip than those without.

In our study, participants without chronic airway diseases had a similar prevalence of sarcopenia (probable and confirmed) to the total global prevalence of $10 \%$ in the healthy older population [4]. However, for participants with chronic airway diseases, our prevalence estimates are slightly higher than previous estimates among COPD subjects $[16,17]$. One explanation for these estimate differences could be that previous studies have reported prevalence of sarcopenia in COPD (range 7.9\% to 8.4\%) using the first EWGSOP1 consensus [30]. Clinical studies have found similar effects in the association between sarcopenia and COPD, but differed in their definition for sarcopenia and cut off values [12]. The updated EWGSOP2 criteria incorporate changes, both conceptually and relating to the diagnostic algorithm [5]. For instance, cut-off values for handgrip and muscle mass were reset, and the central role of gait speed in diagnosing sarcopenia is to discriminate the severity [5]. Therefore, the prevalence of sarcopenia in COPD patients might have been underestimated in the previous studies.

We add to existing knowledge by reporting the prevalence of sarcopenia and its association with chronic airway diseases by using the updated EWGSOP2 criteria [5] and by examining its association with lung function. Additionally, the prevalence of sarcopenia (probable and confirmed) in asthma, COPD and ACO, were similar; hence, we combined and redefined them as chronic airway diseases.

The pathophysiology of secondary sarcopenia is not fully understood. However, similar to primary sarcopenia, it seems that inflammatory processes may lead to metabolic changes, which drive to an

TABLE 6 Odds ratio for chronic airway diseases according to sarcopenia status (EWGSOP2)

\begin{tabular}{|c|c|c|}
\hline & $\begin{array}{c}\text { Probable sarcopenia } \\
\text { OR }(95 \% \mathrm{CI})\end{array}$ & $\begin{array}{c}\text { Confirmed sarcopenia } \\
\text { OR }(95 \% \mathrm{Cl})\end{array}$ \\
\hline No additional adjustment & $1.28(1.02-1.60)$ & $2.13(1.33-3.43)$ \\
\hline \multicolumn{3}{|l|}{ Additionally, adjusted for } \\
\hline $\mathrm{FEV}_{1} / \mathrm{FVC}$ & $1.33(1.01-1.77)$ & $1.40(0.73-2.66)$ \\
\hline Oral corticosteroids use & $1.16(0.92-1.46)$ & $1.94(1.18-3.16)$ \\
\hline Number of comorbidities & $1.25(1.00-1.56)$ & $2.08(1.29-3.35)$ \\
\hline Physical activity ${ }^{\#}$ & $1.27(1.01-1.58)$ & $2.07(1.29-3.33)$ \\
\hline Protein intake $\% \#$ & $1.28(1.02-1.60)$ & $2.15(1.33-3.45)$ \\
\hline \multicolumn{3}{|c|}{$\begin{array}{l}\text { EWGSOP2: European Working Group of Sarcopenia in Older People } 2 ; \mathrm{FEV}_{1} / \mathrm{FVC} \text { : forced expiratory volume } \\
\text { in } 1 \mathrm{~s} / \text { forced vital capacity ratio. All odds are adjusted for age, sex, height, } \% \text { total body fat and smoking } \\
\text { status. \#: based on imputed data, } \mathrm{n}(\%) \text { of missing values: physical activity: } 601 \text { (11.8 protein intake: } 1041 \\
(20.5) .\end{array}$} \\
\hline
\end{tabular}


imbalance between anabolic and catabolic mechanisms. Systemic inflammatory markers, such as interleukin 6 and tumour necrosis factor (TNF)- $\alpha$, C-reactive protein, increase catabolism. Byun et al. [13] found that high sensitivity TNF $\alpha$ (hsTNF $\alpha$ ) correlates with sarcopenia in COPD patients. Indeed, patients with COPD and older asthmatics present with a high-serum level of pro-inflammatory biomarkers [31]. However, for asthma, the underlying role of systemic inflammatory biomarkers in sarcopenia remains yet unknown. Although ageing is another key factor that impacts muscle health, it is not clear whether these changes are associated with chronic airway diseases or are purely due to age. In line with previous studies [15], we observed an increased age-related prevalence of sarcopenia, particularly in those older than 70 years. From our study population (mean age $69.0 \pm 8.8$ years) we can expect an overlap between primary and secondary sarcopenia (in asthma, COPD and ACO). However, in COPD patients, sarcopenia seems to be more disease related than age related. Because muscle dysfunction in COPD is characterised by a shift from type I to type II fibres [32]; whereas, age-related sarcopenia is mainly attributed to muscle denervation and loss of type II fibres which are replaced by adipose tissue [33]. Therefore, these differences indicate that other pathological mechanisms are involved in sarcopenia in COPD, and not explained solely by advanced age. Nevertheless, in asthma patients, the mechanism of primary and secondary sarcopenia is unknown. Consequently, additional longitudinal studies are necessary to further investigate age-related changes in muscle health among persons with chronic airway diseases (i.e. asthma and COPD).

We found that males had slightly higher prevalence of confirmed sarcopenia than females, similar to the sex differences reported previously [34,35]; however, these previous studies focussed only on lean mass prevalence rather than sarcopenia. We showed that the overall prevalence of sarcopenia increased with an increased number of comorbidities. For instance, the total prevalence of probable sarcopenia (10.0\%) can be attributed to a high average of total body fat $(\%)$ in female $(40.1 \pm 6.1)$ and male $(31.0 \pm 5.5)$. Long-term exposure to the fat gain is associated with lower handgrip in older obese [36], and it is known to be one of the essential causes of insulin resistance (another mechanism underlying sarcopenia) [37]. We performed additional adjustment for comorbidities, oral corticosteroids use, physical activity and protein intake; however, all of them had small effects on the magnitude of the estimates. In our study, chronic airway diseases participants with $\mathrm{FEV}_{1}<80 \%$ had lower HGS and lower ASMI compared with $\mathrm{FEV}_{1} \geqslant 80 \%$. These results are consistent with the fact that, in COPD patients, the impairment in lung function affects diverse systems that finally compromise functionality [38].

The strengths of our study are its population-based setting, robust assessment of chronic airway diseases and high-quality information for the diagnosis of sarcopenia (i.e. DXA scan and handgrip). Limitations should be considered when interpreting these results. Firstly, while EWGSOP2 employs the SARC-F questionnaire for case-finding (supplementary 10), we did not use it [39]. Nevertheless, all participants in our study had a measurement of handgrip and DXA scan. Secondly, for the present analysis, we would expect a selection bias due to missing data for walking speed (28.1\%). Mainly because older and severely ill participants could not participate. However, only 2.2\% (32 cases) correspond to the confirmed cases of sarcopenia. Thirdly, as EWGSOP2 does not consider the clinical subgroup with decreased lean mass, normal handgrip and low gait speed as being "sarcopenic", we could have an "unclassified" group. Particularly, in the assessment of COPD patients, who per se have muscle dysfunction, which affects walking speed. Finally, despite the large sample size of the overall population, some subgroup analyses included small numbers (e.g. age group differences (those $<70$ years with confirmed sarcopenia: $\mathrm{n}=13$ ); no comorbidity (those with confirmed sarcopenia: $\mathrm{n}=7)$ ).

In summary, according to the EWGSOP2 recommendations, among older population, probable and confirmed sarcopenia is more prevalent in participants with chronic airway diseases compared with those without. Given the high prevalence of sarcopenia in older people with chronic airway diseases, could be essential routinely screen for handgrip because a diagnosis of "probable sarcopenia" provides an important opportunity for prompt intervention.

Acknowledgements: The authors are grateful for the dedication, commitment and contribution of the citizens, general practitioners and pharmacists of the Ommoord district who took part in the Rotterdam Study.

Support statement: F. Rivadeneira and K. Trajanoska are supported by the Netherlands Scientific Organization (NOW) and ZonMW Project number NW O/ZONMW-VIDI-0 16-136-367.

Conflict of interest: E. Benz has nothing to disclose. K. Trajanoska has nothing to disclose. J.D. Schoufour has nothing to disclose. L. Lahousse reports awards sponsored by AstraZeneca and Chiesi, and expert consultation for Boehringer Ingelheim GmbH and Novartis, outside the submitted work. E.W. de Roos has nothing to disclose. N. Terzikhan has nothing to disclose. C. Medina-Gomez has nothing to disclose. K. Verhamme works for a research group that received/ receives research grants from Yamanouchi, Pfizer/BI, Novartis and GSK, none of which are related to the content of this work. R. Williams has nothing to disclose. B.H. Stricker has nothing to disclose. O.H. Franco has nothing to disclose. M.A. Ikram has nothing to disclose. F. Rivadeneira has nothing to disclose. G. Brusselle has nothing to disclose. 


\section{References}

1 Hughes V. Longitudinal changes in body composition in older men and women: role of body weight change and physical activity. Clin Nutr 2002; 76: 473-481.

2 Keller K, Engelhardt M. Strength and muscle mass loss with aging process. Age and strength loss. Muscles Ligaments Tendons J 2014; 3: 346-350.

3 Cruz-Jentoft AJ, Sayer AA. Sarcopenia. Lancet 2019; 393: 2636-2646.

4 Shafiee G, Keshtkar A, Soltani A, et al. Prevalence of sarcopenia in the world: a systematic review and metaanalysis of general population studies. J Diabetes Metab Disord 2017; 16: 21.

5 Cruz-Jentoft AJ, Bahat G, Bauer J, et al. Sarcopenia: revised European consensus on definition and diagnosis. Age Ageing 2019; 48: 16-31.

6 Ikram MA, Brusselle G, Ghanbari M, et al. Objectives, design and main findings until 2020 from the Rotterdam Study. Eur J Epidemiol 2020; 35: 483-517.

7 Soriano JB, Abajobir AA, Abate KH, et al. Global, regional, and national deaths, prevalence, disability-adjusted life years, and years lived with disability for chronic obstructive pulmonary disease and asthma, 1990-2015: a systematic analysis for the Global Burden of Disease Study 2015. Lancet Respir Med 2017; 5: 691-706.

8 Global Initiative for Asthma. Global Strategy for Asthma Management and Prevention. 2020. Date last accessed: 1 August 2020

9 World Health Organization. Chronic respiratory diseases. Burden of COPD. www.who.int/respiratory/copd/ burden/en/ Date last accessed: 1 August 2020.

10 Global Initiative for Chronic Obstructive Lung disease. Global strategy for the diagnosis, management, and prevention of chronic obstructive pulmonary disease (2020 Report). 2020. https://goldcopd.org/wp-content/ uploads/2019/11/GOLD-2020-POCKET-GUIDE-FINAL-pgsized-wms.pdf Date last accessed: 1 August 2020.

11 Bone AE, Hepgul N, Kon S, et al. Sarcopenia and frailty in chronic respiratory disease. Chron Respir Dis 2017; 14: 85-99.

12 Benz E, Trajanoska K, Lahousse L, et al. Sarcopenia in COPD: a systematic review and meta-analysis. Eur Respir Rev 2019; 28: 190049.

13 Byun MK, Cho EN, Chang J, et al. Sarcopenia correlates with systemic inflammation in COPD. Int J COPD 2017; 12: 669-675.

14 Jones SE, Maddocks M, Kon SSC, et al. Sarcopenia in COPD: prevalence, clinical correlates and response to pulmonary rehabilitation. Thorax 2015; 70: 213-218.

15 Limpawattana P, Inthasuwan P, Putraveephong S, et al. Sarcopenia in chronic obstructive pulmonary disease: a study of prevalence and associated factors in the Southeast Asian population. Chronic Respir Dis 2018; 15: $250-257$

16 Trajanoska K, Schoufour JD, Darweesh SKL, et al. Sarcopenia and its clinical correlates in the general population: the Rotterdam study. J Bone Miner Res 2018; 33: 1209-1218.

17 Borda MG, Celis-Preciado CA, Pérez-Zepeda MU, et al. Sarcopenia in the elderly with a history of COPD/asthma: results of the SABE-Bogotá study. Rev Esp Geriatr Gerontol 2017; 52: 313-316.

18 Ikram MA, Brusselle GGO, Murad SD, et al. The Rotterdam study: 2018 update on objectives, design and main results. Eur J Epidemiol 2017; 32: 807-850.

19 Celli BR, MacNee W, Agusti A, et al. Standards for the diagnosis and treatment of patients with COPD: a summary of the ATS/ERS position paper. Eur Respir J 2004; 23: 932.

20 Quanjer PH, Stanojevic S, Cole TJ, et al. Multi-ethnic reference values for spirometry for the 3-95-yr age range: the global lung function 2012 equations. Eur Respir J 2012; 40: 1324-1343.

21 de Roos EW, Lahousse L, Verhamme KMC, et al. Asthma and its comorbidities in middle-aged and older adults; the Rotterdam Study. Respir Med 2018; 139: 6-12.

22 Lahousse L, Verlinden VJ, van der Geest JN, et al. Gait patterns in COPD: the Rotterdam Study. Eur Respir J 2015; 46: 88-95.

23 Stel VS, Smit JH, Pluijm SM, et al. Comparison of the LASA Physical Activity Questionnaire with a 7-day diary and pedometer. J Clin Epidemiol 2004; 57: 252-258.

24 van der Schaft N, Schoufour JD, Nano J, et al. Dietary antioxidant capacity and risk of type 2 diabetes mellitus, prediabetes and insulin resistance: the Rotterdam Study. Eur J Epidemiol 2019; 34: 853-861.

25 Engberink MF, Bakker SJ, Brink EJ, et al. Dietary acid load and risk of hypertension: the Rotterdam Study. Am J Clin Nutr 2012; 95: 1438-1444.

26 Leening MJ, Kavousi M, Heeringa J, et al. Methods of data collection and definitions of cardiac outcomes in the Rotterdam Study. Eur J Epidemiol 2012; 27: 173-185.

27 van der Willik KD, Ruiter R, van Rooij FJA, et al. Ascertainment of cancer in longitudinal research: the concordance between the Rotterdam Study and the Netherlands Cancer Registry. Int J Cancer 2020; 147: 633-640.

28 Munhoz da Rocha Lemos Costa T, Costa FM, Jonasson TH, et al. Body composition and sarcopenia in patients with chronic obstructive pulmonary disease. Endocrine 2018; 60: 95-102.

29 Lian J, Pan DZ, An XQ, et al. Changes in body composition and associated factors for sarcopenia in patients with chronic obstructive pulmonary disease. Chin Gen Pract 2017; 20: 3504-3508.

30 Cruz-Jentoft AJ, Baeyens JP, Bauer JM, et al. Sarcopenia: European consensus on definition and diagnosis: report of the European Working Group on Sarcopenia in Older People. Age Ageing 2010; 39: 412-423.

31 Barnes PJ. The cytokine network in asthma and chronic obstructive pulmonary disease. J Clin Invest 2008; 118 3546-3556.

32 Maltais F, Decramer M, Casaburi R, et al. An official American Thoracic Society/European Respiratory Society statement: update on limb muscle dysfunction in chronic obstructive pulmonary disease. Am J Respir Crit Care Med 2014; 189: e15-e62.

33 Nilwik R, Snijders T, Leenders M, et al. The decline in skeletal muscle mass with aging is mainly attributed to a reduction in type II muscle fiber size. Exp Gerontol 2013; 48: 492-498.

34 Chung $\mathrm{JH}$, Hwang $\mathrm{HJ}$, Han $\mathrm{CH}$, et al. Association between sarcopenia and metabolic syndrome in chronic obstructive pulmonary disease: the Korea National Health and Nutrition Examination Survey (KNHANES) from 2008 to 2011. COPD 2015; 12: 82-89. 
35 Vermeeren MA, Creutzberg EC, Schols AM, et al. Prevalence of nutritional depletion in a large out-patient population of patients with COPD. Respir Med 2006; 100: 1349-1355.

36 Stenholm S, Sallinen J, Koster A, et al. Association between obesity history and hand grip strength in older adults--exploring the roles of inflammation and insulin resistance as mediating factors. J Gerontol A Biol Sci Med Sci 2011; 66: 341-348.

37 Lombardo M, Boaria A, Aulisa G, et al. Sarcopenic obesity: etiology and lifestyle therapy. Eur Rev Med Pharmacol Sci 2019; 23: 7152-7162.

38 Eisner MD, Iribarren C, Yelin EH, et al. Pulmonary function and the risk of functional limitation in chronic obstructive pulmonary disease. Am J Epidemiol 2008; 167: 1090-1101.

39 Malmstrom TK, Morley JE. SARC-F: a simple questionnaire to rapidly diagnose sarcopenia. J Am Med Dir Assoc 2013; 14: 531-532. 